Volume 19, No 3 International Journal of Radiation Research, July 2021

\title{
Evaluation of water equivalent ratio (WER) values for polyethylene, polymethyl methacrylate, polystyrene, lead, tungsten and aluminum at helium ion energies ranging from 25-250 MeV/u through Monte Carlo simulation
}

\author{
N. Hajiloo ${ }^{*}$, M. Akbari², S. Malekie ${ }^{1}$ \\ ${ }^{1}$ Radiation Application Research School, Nuclear Science and Technology Research Institute, Karaj, Iran \\ ${ }^{2}$ Pars Isotope Co., P.O. Box 14376-63181, Karaj, Iran
}

(2)

- Original article

*Corresponding authors:

Nahid Hajiloo, Ph.D., E-mail: nhajiloo@aeoi.org.ir

Revised: July 2020

Accepted: July 2020

Int. J. Radiat. Res., July 2021; 19(3): 661-668

DOI: $10.29252 /$ ijrr.19.2.661

\begin{abstract}
Background: There is no data concerning water equivalent ratio (WER) values for helium ion beams in an extensive range of energies as well as relevant dosimetric materials. Materials and Methods: In this work, quantities related to depth-dose profiles and WER values were evaluated in water, Polyethylene (PE), Polymethyl Methacrylate (PMMA), Polystyrene (PS), Lead $(\mathrm{Pb})$, Tungsten $(\mathrm{W})$ and Aluminum (Al) for helium ion energies ranging from 25-250 MeV/u using MCNPX 2.4.0 Monte Carlo code. Results: For all the studied energy range, the mean values of WER for PMMA, PE, PS, Pb, W and Al were 1.161, 0.995, 1.049, 5.421, 9.512 and 2.091, respectively. Among the studied materials, PE and $\mathrm{W}$ showed the least and most difference to water, respectively. Also the WER values of some of the studied materials for helium ion beams were compared with the same materials for proton beam. Conclusion: The evaluated WER values were in acceptable accordance with the data reported in the literature by less than $2.6 \%$ difference. Also, WER values of the mentioned materials for helium ions and protons have been compared and it was concluded that dose characteristics of PE are the most similar to water in the field of both helium ions and proton beams.
\end{abstract}

Keywords: Helium ion beam, dosimetry materials, water equivalent ratio.

\section{INTRODUCTION}

Discrepancies in interaction mechanisms address therapeutic differences using either photon or hadron beams in radiation therapy. In photon radiation therapy, in addition to the tumor, healthy tissues also receive noticeable dose before and after the target volume. For deep-seated tumors, since photons should travel deeper depths inside the body, this unwanted dose is higher, which increases the possibility to induce the secondary cancer incidence (1-4). In 1946, for the first time, Wilson proposed to use high-energy ions in radiation therapy (5). He recommended that specific ionization properties of heavy particles are usable in clinical and biological applications (5-7).

Ion therapy exhibits a wide range of potential applications, namely: pediatric tumors like medulloblastoma, Rhabdomyosarcoma, ependymomas, gliomas, and craniopharyngiomas, and also central nervous system tumors called glioblastomas (8).

When a high energy ion beam enters the tissue, along with the path, at first, its speed is almost constant and has a minimum energy loss. At the end of the path, while the charged particle is going to stop, it suddenly loses maximum dose at a short distance, this is called as the Bragg Peak. Due to the presence of the Bragg peak in ion depth-dose profile, there is potential capability of delivering the dose to the tissues at 
a given region and eliminate dose to the tissues before and after the target region (9).

Currently, the use of proton, helium, carbon, and oxygen ions is expanding, and each of them has its advantages and disadvantages. Because of the physical and radiobiological properties of helium ions compared to the most clinically available ion beams (i. e., proton and carbon), such as beam sharpness and less fragment, currently in the Heidelberg Ion-Beam Therapy (HIT) Center, alongside other ions, research on the use of helium ions in treatment is conducted $(2,10-12)$. For tumor entities which do not necessarily require an elevated linear energy transfer (LET) and associated relative biological effectiveness (RBE) of heavy ions, helium ions delivered with state-of-the-art techniques have the potential to improve clinical outcome in comparison to the more broadly deployed proton beams. Helium ions with reduced lateral straggling in comparison to protons can reduce dose of healthy tissues and increase delivered dose to the tumor. In terms of radiobiological effects, helium ions have high LET and correspondingly high RBE and also low oxygen enhancement ratio (OER) in comparison to the protons. Helium ions have a shorter fragmentation tail after Bragg peak than in heavier ions and, therefore they deliver low distal dose to target. Considering the mentioned reasons, treatment using helium ions can be an alternative option in ion therapy that provides increase in dose delivery because of reduction in their penumbra in comparison to protons and because of less fragmentation tail than in heavier ions such as carbon ions. Also in terms of Physic aspect, helium ions are better than protons for spot scanning method due to better control of slot spot dimensions. In particular, helium ion therapy can reduce dose effectively in organs at risk that are located close to target. They also can reduce the whole dose to the body that is related to risk of radiation-induced secondary cancer $(13,14)$. Dose distribution data in radiation therapy is generally obtained from measurements in phantoms that are made of tissue equivalent materials, and it is rarely possible to measure the dose distribution in the patient's body, in any type of radiation therapy.
Commonly, for dose distribution measurements, water phantom is used as a tissue equivalent material. Since using water phantom has some disadvantages, especially if used with a dosimeter, many solid water equivalent phantoms have been introduced for dose distribution measurements (15).

For determining penetration range, water equivalent ratio (WER) or water equivalent thickness (WET) is mostly used. Since for wide range of energies, WER and WET are not accessible for various phantoms and dosimetry materials. Therefore some researchers have provided experimental or calculation results for WER and WET values (6,16-19). However, WER values for protons exist even in upper and lower range rather than the therapeutic energy range, and there is enough experimental and analytical data in the therapeutic energy range $(6,15,16,20$-24). But, to the best of the authors' knowledge, for helium ions, except for some analytical calculations, there is no experimental data and even simulation calculation results. Therefore, the main motivation for the calculations that were presented in this work was the lack of data for dose evaluations in helium ion beam therapy. For this reason, in this work, WER values were calculated through MCNP Monte Carlo simulation code at low and medium energy range $(25-250 \mathrm{MeV} / \mathrm{u})$ of helium ions for various relevant potential materials that are applied in clinical practice.

\section{MATERIALS AND METHODS}

\section{Water equivalent ratio}

The range of ions (R) is described as the depth of absorbent material whereby the particle beam loses $80 \%$ of its maximum energy after the Bragg peak. In other words, $\mathrm{R}$ is obtained as the depth distal to the Bragg peak where the dose reduces to $80 \%$ of its highest value at the Bragg peak $\left(\mathrm{R}^{\mathrm{w}} 80 \%\right)^{(17)}$.

In the clinical practice, the water equivalent ratio (WER) is applied to determine the ratio of the range of the ion beam in water $\left(\mathrm{R}^{\mathrm{w}} 80 \%\right)$ to the range of ion beam in a specified material $m$ $\left(\mathrm{R}^{\mathrm{m}}\right.$ 80\%). WER is defined as below (17): 
$W E R=\frac{R^{W_{8096}}}{R^{m_{8096}}}$

In the present research, WER is calculated using eq. 1 , in low and medium energies of helium ion beams in water, Polyethylene (PE), Polymethyl Methacrylate (PMMA), Polystyrene (PS), Lead $(\mathrm{Pb})$, Tungsten $(\mathrm{W})$ and Aluminum (Al), using the Monte Carlo simulation. Considered densities for studied materials are 1 , $0.94,1.19,1.06,11.34,19.30$ and $2.70 \mathrm{~g} / \mathrm{cm}^{3}$ for water, $\mathrm{PE}, \mathrm{PMMA}, \mathrm{PS}, \mathrm{Pb}, \mathrm{W}$ and $\mathrm{Al}$ respectively (25).

\section{Monte Carlo assessments}

In hadron therapy, the MCNP code is used as a non-deterministic and accurate code for evaluation of dosimetric characteristics of the charged particles. Therefore, it is used for different areas of particle therapy such as calculating dose distributions in tissues or phantoms as well as WET and WER values $(16,17)$.

In this theoretical work, the MCNPX 2.4.0 code is applied for helium ion transportation and dose distribution calculations to assess WER values for mentioned materials. Mesh tallies have been used to dose calculations along the beam. The Mesh Tally is a method to calculate particle flux, dose, or other quantities on a rectangular, cylindrical, or spherical grid overlaid on top of the standard problem geometry. Particles are tracked through the independent mesh as part of the regular transport problem, and the contents of each mesh cell are written to a file at the end of the problem. In the present work the third type of Mesh Tally scores energy deposition data in which the energy deposited per unit volume from all particles is included (25). A cylindrical phantom containing water with dimensions of $1000 \mathrm{~mm}$ in height and $300 \mathrm{~mm}$ in diameter was simulated. A circular cross section beam with 2 $\mathrm{mm}$ diameter helium ion mono-energetic beam is incident perpendicular to the phantom surface. By defining mesh-tallies in the input file, 10000 cylindrical detectors with dimensions of $1 \mathrm{~mm}$ in height and $20 \mathrm{~mm}$ in diameter were considered along with the beam path in the water for calculating helium deposited energy in each detector. Figure 1 shows the details of the supposed geometry.
In the MCNPX code, as recommended in its manual, the maximum energy parameter (Emax) of helium physics option was set to system's energy max plus $5 \mathrm{MeV}$ (26). Different physics models are used in this code such as ISABEL, CEM, and Bertini modes for energies above 150 $\mathrm{MeV}$ (37.5 MeV/u in this work). For simulations of the helium interactions $>150 \mathrm{MeV}$, the LCA card with different physic models was used. WER value assessment for the studied materials was performed in the range of $25-250 \mathrm{MeV} / \mathrm{u}$ by the step of $12.5 \mathrm{MeV} / \mathrm{u}$. Depth distal to the Bragg peak, in which the dose reduces to $80 \%$ of its highest value at the Bragg peak, namely clinical range (Rw80\%), is extracted from the depth-dose profiles in each energy. Same steps were done with other studied materials. In other words, by changing water phantom material to PMMA, PS, PE, Pb, W and, Al, dose distribution in the detectors was calculated and, the depth dose profile was obtained in each energy, afterward, clinical ranges of helium ions with different energies were extracted from beam profiles for each considered material. By considering Rm80\% for each material and Rw80\% for water and using eq. (1), WER was calculated. Statistical relative errors were less than $1 \%$ in all simulation calculations.

According to the eq. (1), the calculated values of WER depend on the amount of alpha particle range in the water and subject solid matter. The uncertainty in the calculation of the alpha particle range leads to the uncertainty in the WER calculation. Since the range values are obtained from the depth-dose curves, the uncertainty in the calculation of the range might be affected by the uncertainties in the calculation of the dose in each computational voxel (mesh). In the Monte Carlo calculations, $10^{6}$ particle histories were tracked to reduce the relative error in the dose calculation to less than $1 \%$ in each mesh. Excel software (version 2010) was used for data analysis and graphing. Linear interpolation of dose values was used to calculate more accurate range values. According to the mesh dimensions used in this study, the systematic uncertainty in calculating the alpha particle range is $1 \mathrm{~mm}$, which is acceptable for the ion beam applications in therapeutic and dosimetry purposes. 


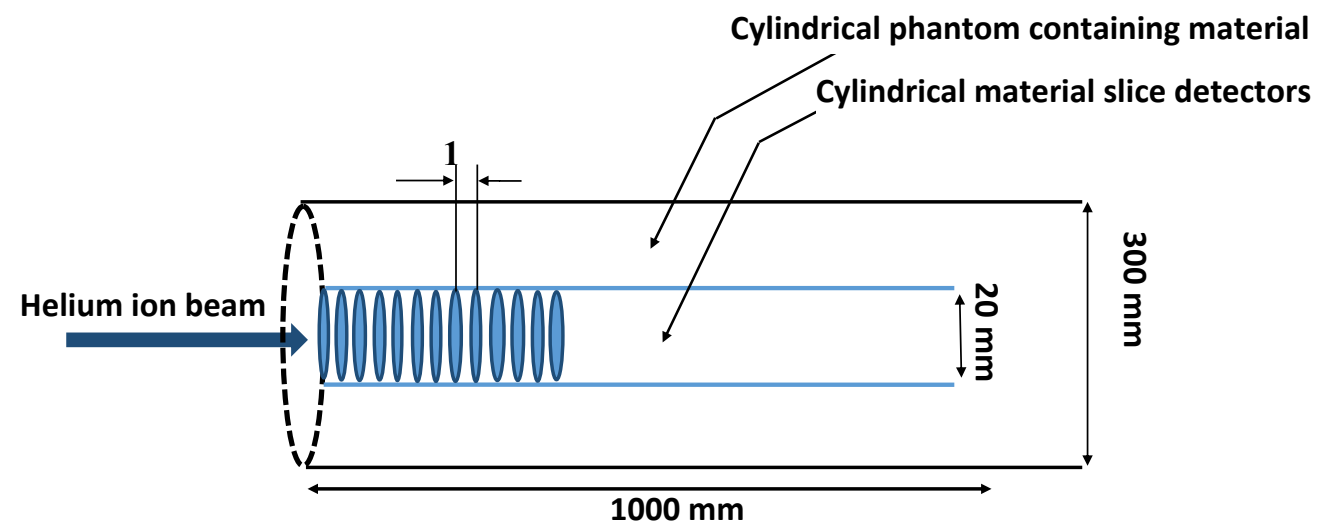

Figure 1. Schematic diagram of the simulated geometry to calculate dose distribution, using MCNPX code. The phantom contains studied materials including PMMA, PS, PE, Pb, W and, Al.

\section{RESULTS}

Depth-dose profiles of helium ions in the water, PMMA, PS, PE, $\mathrm{W}, \mathrm{Pb}$ and, $\mathrm{Al}$ were obtained using MCNP code calculations. Depth-dose profiles in the mentioned materials, in intermediate energy of helium ions (150 $\mathrm{MeV} / \mathrm{u}$ ) are given in Fig. 2. As can be seen, the Bragg peak depth is different in each of the studied materials. The Bragg peak positions for PE and PS are located on the sides of water's Bragg peak position and PE is the closest to water. While the peak position of metals has the greatest distance from the water.

WER values were extracted from MCNP calculations by evaluating $\mathrm{R}^{\mathrm{w}} 80 \%$ and $\mathrm{R}^{\mathrm{m}} 80 \%$ from the beam profiles of the mentioned materials at different energies using equation (1) and are listed in table 1. For all the studied energy ranges, the mean values of WER for PMMA, PE, PS, Pb, W and Al were 1.161, 0.995, 1.049, 5.421, 9.512 and, 2.091, respectively. Therefore, $\mathrm{W}$ has the biggest WER value among the studied materials and PE exhibits the least difference compared to water. In addition, the WER values of the materials whose densities are close to that of water are almost constant by increasing energy, whereas in high-density materials such as $\mathrm{W}, \mathrm{Al}$, and $\mathrm{Pb}$ the WER values increase by increasing energy. Increases for these materials are $0.103,0.657$, and 1.046 respectively. For a better representation, WER values of the studied materials were compared in figure 3. As can be seen, PE is closer to water than five other materials. In table 1 and figure 3 , a comparison between evaluated WER values and the reported results published in the literature is exhibited. As can be observed, the evaluated WER values are in good agreement with the reported data (6). The maximum difference in WER values between results from MCNP calculations and obtained data from the literature was about $2.6 \%$.

To evaluate the feasibility of applying the studied dosimetric materials for both proton and helium ion beams, especially for centers that are trying to derive a benefit from the advantages of helium ion beams besides the proton (2), the WER values of some of the studied materials for helium ion beams were compared with the same materials for proton utilization scenario. This comparative study can help us to understand whether the potential dosimetric materials which can be applied for helium ion beam dosimetry, could be used in proton dosimetry. In the case of PMMA, a 1.16 average value is obtained for both proton and helium ion beams. For PE, 1.0001 and 1.002 average values are calculated from table 2 for helium and proton beams, respectively. In the case of PS, a 1.05 value is extracted for both the ion beams. For Al, values of 2.08 and 2.12 are obtained for helium and proton beams, respectively. The results indicate that by changing the ion beams between proton and helium, in the range of the investigated energies in table 2 , WER values do not have important change (maximum less than $7.5 \%$, which is relevant to PE material). PMMA and PS have the same average WER value for the investigated energy range in table 2 .

Int. J. Radiat. Res., Vol. 19 No. 3, July 2021 


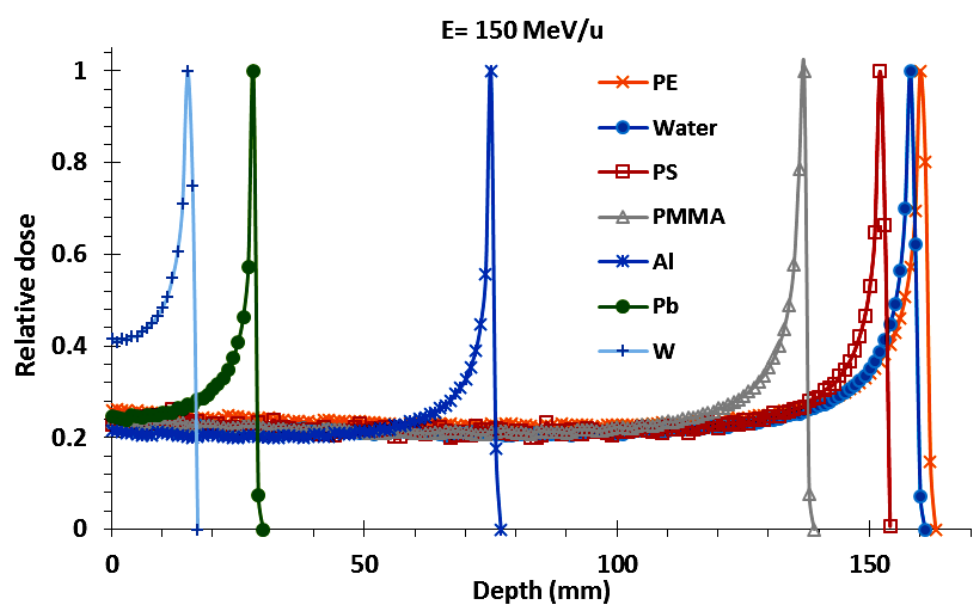

Figure 2. Depth-dose profiles in the studied materials for $150 \mathrm{MeV} / \mathrm{u}$ helium ion energy (calculated by MCNP code).

Table 1. The calculated WER values (this work) for different energies of helium ions in some of the studied materials compared with the literature ${ }^{(6)}$

\begin{tabular}{|c|c|c|c|c|c|c|c|c|c|c|c|c|}
\hline \multirow{3}{*}{$\mathrm{E}(\mathrm{MeV} / \mathrm{u})$} & \multicolumn{12}{|c|}{ ER } \\
\hline & \multicolumn{3}{|c|}{ PMMA } & \multirow{2}{*}{\begin{tabular}{|c|} 
PE \\
This work
\end{tabular}} & \multirow{2}{*}{\begin{tabular}{|c|} 
PS \\
This work \\
\end{tabular}} & \multicolumn{3}{|c|}{$\mathrm{Pb}$} & \multirow{2}{*}{\begin{tabular}{|c|} 
W \\
This work
\end{tabular}} & \multicolumn{3}{|c|}{ Al } \\
\hline & This work & $\operatorname{Ref}^{(6)}$ & $\% \Delta_{\text {WER }}$ & & & This work & $\mid \operatorname{Ref}^{(6)}$ & $\% \Delta_{\text {WER }}$ & & This work & $\operatorname{Ref}^{(6)}$ & $\% \Delta_{\text {WER }}$ \\
\hline 25.0 & 1.166 & - & sing & 1.012 & 1.105 & 5.101 & - & ons & 8.789 & 2.003 & - & - \\
\hline 37.5 & 1.157 & - & - & 1.001 & 1.071 & 5.042 & - & - & 8.890 & 2.018 & - & - \\
\hline 50.0 & 143 & - & - & 0.996 & 1.043 & 124 & - & - & 9.188 & 2.016 & - & - \\
\hline 62.5 & 1.168 & - & - & 0.997 & 1.032 & 5.167 & - & - & 9.189 & 2.098 & - & - \\
\hline 75.0 & 1.172 & - & - & 0.999 & 1.043 & 5.233 & - & - & 9.302 & 2.106 & 2.068 & -1.84 \\
\hline 87.5 & 157 & - & - & 0.984 & 1.037 & 5.308 & - & - & 9.395 & 2.108 & 2.082 & -1.25 \\
\hline 100.0 & 163 & - & - & 0.988 & 1.040 & 5.336 & - & & 9.497 & 2.050 & 2.089 & 1.87 \\
\hline 112.5 & 1.166 & - & - & 0.989 & 1.047 & 5.412 & 5.426 & 0.26 & 9.508 & 2.093 & 2.094 & 0.05 \\
\hline 125.0 & 166 & - & & 0.990 & 1.044 & 5.420 & 5.487 & 1.22 & 9.522 & 2.081 & 2.098 & 0.81 \\
\hline 137.5 & 154 & 1.158 & 0.35 & 0.988 & 1.039 & 5.434 & 5.532 & 1.77 & 9.549 & 2.075 & 2.101 & 1.24 \\
\hline 150.0 & 1.159 & 1.158 & -0.09 & 0.989 & 1.039 & 5.470 & 5.568 & 1.76 & 9.446 & 2.096 & 2.103 & 0.33 \\
\hline 162.5 & & 1.158 & 0.09 & 0.988 & 1.045 & 5.518 & 5.599 & 1.45 & 9.550 & 2.110 & 2.106 & -0.19 \\
\hline & & 1.158 & -0.60 & 0.987 & 1.048 & 5.494 & 5.626 & 2.35 & 9.779 & 2.113 & 2.108 & -0.24 \\
\hline 187.5 & 1.155 & 1.158 & 0.26 & 0.988 & 1.049 & 5.505 & 5.651 & 2.58 & 9.812 & 2.140 & 2.110 & -1.42 \\
\hline 200 & 1.158 & 1.158 & 0.00 & 0.974 & 1.056 & 5.701 & 5.674 & -0.48 & 9.695 & 2.153 & 2.111 & -1.99 \\
\hline 212.5 & 167 & 1.157 & -0.86 & 0.998 & 1.050 & 5.577 & 5.694 & 2.05 & 9.878 & 2.131 & 2.113 & -0.85 \\
\hline 225 & & 1.157 & -0.17 & 1.082 & 1.044 & 5.781 & 5.714 & \begin{tabular}{|l|}
-1.17 \\
\end{tabular} & 9.911 & 2.120 & 2.114 & -0.28 \\
\hline 237.5 & 1.159 & 1.157 & -0.17 & 0.979 & 1.047 & 5.758 & 5.732 & -0.45 & 9.835 & 2.106 & 2.116 & 0.47 \\
\hline 250 & 1.167 & 1.157 & -0.86 & 0.983 & 1.048 & 5.627 & 5.749 & 2.12 & 10.001 & 2.117 & 2.117 & 0.00 \\
\hline
\end{tabular}

Table 2. Comparison between WER values for helium ions (this work) and protons $(12,15)$ for some of the studied materials.

\begin{tabular}{|c|c|c|c|c|c|c|c|c|c|c|c|c|}
\hline \multirow{3}{*}{$\mathrm{E}(\mathrm{MeV} / \mathrm{u})$} & \multicolumn{12}{|c|}{ WER } \\
\hline & \multicolumn{2}{|c|}{ PMMA } & \multicolumn{3}{|c|}{ PE } & \multicolumn{3}{|c|}{ PS } & \multicolumn{3}{|c|}{ Al } & \multirow[b]{2}{*}{$\% \Delta_{\text {WER }}$} \\
\hline & Helium & Proton & $\% \Delta_{\text {WER }}$ & Helium & Proton & $\% \Delta_{\text {WER }}$ & Helium & Proton & $\% \Delta_{\text {WER }}$ & Helium & Proton & \\
\hline 25.0 & 1.166 & - & - & 1.012 & - & - & 1.105 & 1.044 & 5.5 & 2.003 & - & - \\
\hline 50.0 & 1.143 & - & - & 0.996 & - & - & 1.043 & - & - & 2.016 & 2.066 & -2.5 \\
\hline 100.0 & 1.163 & 1.158 & 0.7 & 0.988 & 1.002 & -1.4 & 1.040 & - & - & 2.050 & 2.102 & -2.5 \\
\hline 125.0 & 1.166 & - & - & 0.990 & 1.003 & -1.3 & 1.044 & 1.045 & -0.1 & 2.081 & 2.120 & -1.8 \\
\hline 150.0 & 1.159 & 1.158 & 0.1 & 0.989 & 1.002 & -1.3 & 1.039 & 1.045 & -0.6 & 2.096 & 2.108 & -0.6 \\
\hline 175 & 1.165 & - & - & 0.987 & 1.001 & -1.4 & 1.048 & 1.044 & 0.4 & 2.113 & 2.132 & 0.9 \\
\hline 200 & 1.158 & 1.157 & 0.1 & 0.974 & 1.001 & -2.8 & 1.056 & 1.044 & 1.1 & 2.153 & 2.131 & -1.2 \\
\hline 225 & 1.159 & - & - & 1.082 & 1.001 & 7.5 & 1.044 & - & - & 2.120 & 2.141 & -0.9 \\
\hline 250 & 1.167 & - & - & 0.983 & 1.001 & -1.8 & 1.048 & - & - & 2.117 & 2.133 & -0.7 \\
\hline
\end{tabular}

Int. J. Radiat. Res., Vol. 19 No. 3, July 2021 


\section{DISCUSSION}

As it is well-known, water is the most reliable and applicable material instead of biological tissues in dosimetry of ion beams (16). Some plastic materials, containing PMMA, PE, PS can be applied instead of water for dosimetry purposes. Moreover, some of the metallic materials, including $\mathrm{Al}, \mathrm{W}$, and $\mathrm{Pb}$ are used as the components of the beam line or dosimeters (23, 27-28). WER values can be applied as a conversion factors to convert dose in mentioned above solid dosimetric materials to dose in water. For some of the indicated above materials, WER values were determined and have been reported in limited energy ranges. For PMMA, in the energy range of $137.5 \mathrm{MeV} / \mathrm{u}$ to $250 \mathrm{MeV} / \mathrm{u}$, WER values were found in the references, while in the range of $75 \mathrm{MeV} / \mathrm{u}$ to $125 \mathrm{MeV} / \mathrm{u}$ the authors couldn't find any data in the literatures. Furthermore, for $\mathrm{Pb}$ and $\mathrm{Al}, \mathrm{WER}$ values have been reported for limited energy ranges (6). Due to lack of data for WER values of PE, PS, and W, in this work, WER values of these materials have been calculated. Besides that, for PMMA, Al and $\mathrm{Pb}$, the WER values calculated for extended energy ranges, which are not available in the published literatures.

The mean differences of the calculated WER values of PMMA, $\mathrm{Al}$ and $\mathrm{Pb}$ in this study compared to the reported results are $0.34 \%$, $0.85 \%$ and $1.78 \%$, respectively and the maximum difference was about $2.6 \%$ relevant to $\mathrm{Pb}$ for the helium energy of $187.5 \mathrm{MeV} / \mathrm{u}$ indicating acceptable agreement to the published literature ${ }^{6}$ ).

The results of this research indicate that WER values of high density materials increase by increasing helium ion energy. WER values of $\mathrm{Al}$, $\mathrm{Pb}$ and $\mathrm{W}$ vary by energy variations and, the highest is relevant to $\mathrm{W}$, which changes from 8.789 to 10.001 by energy variations ranging from $25 \mathrm{MeV} / \mathrm{u}$ to $250 \mathrm{MeV} / \mathrm{u}$. This phenomenon was not seen in the results relevant to PMMA, PS and, PE. For helium ion beams, the WER values were largely independent of energy for low density materials. This independency to the energy was reported in the published literature for carbon and proton beams transporting in low density materials ${ }^{6}$, 16).

According to the data presented in table 2, the dosimetric similarity of PMMA to water at $100 \mathrm{MeV} / \mathrm{u}, 150 \mathrm{MeV} / \mathrm{u}$ and $200 \mathrm{MeV} / \mathrm{u}$ protons and helium ion energies is not considerably dependent on the ion type. In the case of PE material, the dosimetric similarity to water depends notably on the type of ion being studied. In this material, the values of WER change from $1.3 \%$ to $7.5 \%$ by changing the type of ion investigated. Furthermore, in the case of PS material, the change in dosimetric similarity to water in the studied and compared energy range is not importantly dependent on the type of ion. For Al material, a maximum of $2.5 \%$ difference in WER values with ion type change indicates that the ion type change importantly alters the dosimetric similarity of $\mathrm{Al}$ material to water.

In ion therapy centers (for instance, the Heidelberg Ion Therapy Center (HIT) (2)) that plan to use all three carbon, proton and helium ions for treatment, choosing the best phantom material that most closely resembles water in terms of dosimetry for the therapeutic range is one of the challenges ahead. It is very important in terms of reducing set-up time and reducing the cost of providing phantoms of different materials for each type of ion. Investigating the parameters of dosimetric similarity of a much wider range of materials to water in the therapeutic energies of all three ions can be used to select a material that can be applied for all three ions leads to save the time of measurement, quality assurance and cost significantly.

\section{CONCLUSION}

In summary, in low and high density materials WER values were smaller and larger relative to water respectively. Also WER values of high density materials increase by increasing helium ion energies, this phenomenon is not seen in the low density materials. In addition, it can be concluded that the WER values do not have important changes for proton and helium

Int. J. Radiat. Res., Vol. 19 No. 3, July 2021 
ions in the same energies.

\section{Conflicts of interest: Declared none.}

\section{REFERENCES}

1. Yajnik S (2012) Proton Beam Therapy: How Protons are Revolutionizing Cancer Treatment. Springer Science \& Business Media.

2. Tessonnier T, Mairani A, Chen W, Sala P, Cerutti F, Ferrari A, Haberer T, Debus J, Parodi K (2018) Proton and helium ion radiotherapy for meningioma tumors: a Monte Carlobased treatment planning comparison. Radiation Oncology, 13: 2 .

3. Hong L, Goitein M, Bucciolini M, Comiskey R, Gottschalk B, Rosenthal S, Serago C, Urie M (1996) A pencil beam algorithm for proton dose calculations. Physics in Medicine \& Biology, 41: 1305.

4. Bauer J, Unholtz D, Kurz C, Parodi K (2013) An experimental approach to improve the Monte Carlo modelling of offline $\mathrm{PET} / \mathrm{CT}$-imaging of positron emitters induced by scanned proton beams. Physics in Medicine \& Biology, 58: 5193.

5. Wilson R (1946) Radiological use of fast protons. Radiology, 47: 487-491.

6. Zhang R, Taddei PJ, Fitzek MM, Newhauser WD (2010) Water equivalent thickness values of materials used in beams of protons, helium, carbon and iron ions. Physics in Medicine \& Biology, 55: 2481

7. Schulz-Ertner D and Tsujii H (2007) Particle radiation therapy using proton and heavier ion beams. Journal of clinical oncology, 25: 953-964.

8. Veiskarami A, Sadeghi M,Sardari D, Malekie S (2019) Optimization of beamline diameter in spot scanning proton therapy for minimization of secondary particles using fininte element method. Radiation Physics and Engineering, 1: 1-7.

9. Newhauser WD and Zhang R (2015) The physics of proton therapy. Physics in Medicine \& Biology, 60: R155.

10. Tessonnier $T$, Böhlen $T$, Ceruti F, Ferrari A, Sala P, Brons $S$, Haberer T, Debus J, Parodi K, Mairani A (2017) Dosimetric verification in water of a Monte Carlo treatment planning tool for proton, helium, carbon and oxygen ion beams at the Heidelberg ion beam therapy Center. Physics in Medicine \& Biology, 62: 6579.

11. Dokic I, Mairani A, Niklas M, Zimmermann F, Chaudhri N, Krunic D, Tessonnier T, Ferrari A, Parodi K, Jäkel O (2016) Next generation multi-scale biophysical characterization of high precision cancer particle radiotherapy using clinical proton, helium-, carbon-and oxygen ion beams. Oncotarget, 7: 56676.

12. Mairani A, Dokic I, Magro G, Tessonnier T, Kamp F, Carlson D, Ciocca M, Cerutti F, Sala P, Ferrari A (2016) Biologically optimized helium ion plans: calculation approach and its in vitro validation. Physics in Medicine Biology, 61: 4283.
13. Lühr A, Hansen D C, Teiwes R, Sobolevsky N, Jäkel O, Bassler N (2012) The impact of modeling nuclear fragmentation on delivered dose and radiobiology in ion therapy. Physics in Medicine Biology, 57: 5169.

14. Horst F, Schuy C, Weber U, Brinkmann K T, Zink K (2017). Measurement of charge-and mass-changing cross sections for He $4+\mathrm{C} 12$ collisions in the energy range $80-220 \mathrm{MeV} /$ $\mathrm{u}$ for applications in ion beam therapy. Physical Review $C$, 96: 024624.

15. Akbari MR, Yousefnia H, Mirrezaei E (2014) Calculation of water equivalent ratio of several dosimetric materials in proton therapy using FLUKA code and SRIM program. Applied Radiation and Isotopes, 90: 89-93.

16. Safigholi $H$ and Song $W$ Y (2018) Calculation of water equivalent ratios for various materials at proton energies ranging $10-500 \mathrm{MeV}$ using MCNP, FLUKA, and GEANT4 Monte Carlo codes. Physics in Medicine \& Biology, 63: 155010.

17. Newhauser $\mathrm{W}$, Fontenot J, Zheng $\mathrm{Y}$, Polf J, Titt $\mathrm{U}$, Koch $\mathrm{N}$, Zhang X, Mohan R (2007) Monte Carlo simulations for configuring and testing an analytical proton dosecalculation algorithm. Physics in Medicine \& Biology, 52: 4569.

18. Newhauser W (2001) Proton field calibration measurements and analysis with the calibration setup program (CSU) version 1.1. Massachusetts General Hospital Report.

19. Bagheri R, Moghaddam AK, Azadbakht B, Akbari MR, Shirmardi SP (2019) Determination of water equivalent ratio for some dosimetric materials in proton therapy using MNCPX simulation tool. Nuclear Science and Techniques, 30: 31 .

20. Zhang R, Newhauser W D (2009) Calculation of water equivalent thickness of materials of arbitrary density, elemental composition and thickness in proton beam irradiation. Physics in Medicine \& Biology, 54: 1383.

21. Lee $C$, Lee $Y$, Tung $C$, Cheng $H$, Chao T (2014) MCNPX simulation of proton dose distribution in homogeneous and CT phantoms. Radiation Physics and Chemistry, 95: 302-304.

22. Fellin F, Righetto R, Fava G, Trevisan D, Amelio D, Farace $P$ (2017) Water equivalent thickness of immobilization devices in proton therapy planning-Modelling at treatment planning and validation by measurements with a multilayer ionization chamber. Physica Medica, 35: 31-38.

23. Lourenço A, Shipley D, Wellock N, Thomas R, Bouchard $H$, Kacperek A, Fracchiolla F, Lorentini S, Schwarz M, MacDougall N (2017) Evaluation of the water-equivalence of plastic materials in low-and high-energy clinical proton beams. Physics in Medicine \& Biology, 62: 3883.

24. de Vera P, Abril I, Garcia-Molina R (2014) Water equivalent properties of materials commonly used in proton dosimetry. Applied Radiation and Isotopes, 83: 122-127.

25. Berger M, Inokuti M, Andersen H, Bichsel H (1993) Stopping powers and ranges for protons and alpha particles. ICRU Report 49.

26. Los Alamos National Laboratory (2002). Monte Carlo NParticle Transport Code System for Multiparticle and High Energy Applications MCNPX' 2.4.0. Los Alamos, New Mexico. 
27. Martišíková M, Gehrke T, Berke S, et al. (2018) Helium ion beam imaging for image guided ion radiotherapy. Radiation Oncology, 13: 109.

28. Volz L, Kelleter L, Brons S, et al. (2020) Experimental exploration of a mixed helium/carbon beam for online treatment monitoring in carbon ion beam therapy. Physics in Medicine \& Biology, 65: 055002.

29. Leverington BD, Dziewiecki M, Renner L, Runze R (2017) A Scintillating Fibre Beam Profile Monitor for Ion Therapy Beams. Journal of Instrumentation, 13: P05030.
30. Kanematsu N, Koba Y, Ogata R (2013) Evaluation of plastic materials for range shifting, range compensation, and solid -phantom dosimetry in carbon-ion radiotherapy. Medical Physics, 40: 041724.

31. Mónica A (2017) Water-equivalence of phantom materialsin proton and carbon-ion dosimetry. PhD Thesis, University College London.

32. Charlie Ma C-M and Lomax T (2013) Proton and Carbon Ion Therapy. CRC Press, Taylor \& Francis Group. 